\title{
Redécouvrir les travaux du Design Methods
}

Movement

Jodelle Zetlaoui-Léger

\section{OpenEdition}

Édition électronique

URL : http://journals.openedition.org/crau/499

DOI : $10.4000 /$ crau.499

ISSN : 2547-5746

Éditeur

Éditions du patrimoine

Édition imprimée

Date de publication : 1 septembre 2013

Pagination : $57-70$

ISBN : 978-2-7577-0109-6

ISSN : 1296-4077

Référence électronique

Jodelle Zetlaoui-Léger, «Redécouvrir les travaux du Design Methods Movement », Les Cahiers de la recherche architecturale et urbaine [En ligne], 28 | 2013, mis en ligne le 12 septembre 2017, consulté le 19 avril 2019. URL : http://journals.openedition.org/crau/499; DOI : 10.4000/crau.499 
La prise en compte des enjeux environnementaux et de développement durable dans la production architecturale et urbaine induisent des transformations importantes dans les démarches de projet et les cultures professionnelles. Ces nouvelles situations d'exercice font écho aux préoccupations exprimées par des théoricienspraticiens anglo-saxons du Design Methods Movement au cours des années 1960 à 2000. S'interrogeant sur les fondements du processus de conception, ces auteurs ont été conduits à considérer celui-ci dans une acception large, en y intégrant des dynamiques évaluatives et participatives au nom de valeurs et de principes qui sont aujourd'hui ceux du développement durable. Cet article vise à donner un aperçu des travaux de ce mouvement assez méconnu en France et à montrer en quoi, devant les interrogations que pose la poursuite d'objectifs de développement durable à l'activité de conception, ils mériteraient d'être (re)découverts.

\section{Redécouvrir les travaux du Design Methods Movement}

JODELLE ZETLAOUI-LÉGER

Depuis le milieu des années 2000, plusieurs travaux de recherche se référant à différents contextes européens suggèrent que la prise en compte des enjeux environnementaux et de développement durable dans la production architecturale et urbaine conduirait à introduire des transformations, voire une "rupture paradigmatique » dans les procès de projet et les cultures professionnelles ${ }^{1}$. Lors d'une recherche récente ${ }^{2}$ portant sur la fabrication des premières opérations d'écoquartiers en France, nous avons pu prendre la mesure de ce phénomène en nous intéressant à la façon dont "l'impératif participatif » accompagnant aujourd'hui de telles opérations pouvait bousculer les pratiques habituelles des acteurs de l'aménagement de la construction. Nous avons en particulier constaté que si dans près des trois-quarts des opérations étudiées, le stade consultatif peinait encore à être dépassé, la préoccupation d'accompagner les changements sociétaux et les évolutions technologiques liés à la prise de considération des enjeux de développement durable ${ }^{3}$ a incité une majorité de collectivités à

1. Cyria Emelianoff, « Les quartiers durables en Europe : un tournant urbanistique?", Urbia. Les Cahiers du développement urbain durable, $n^{\circ} 4$, juin 2007, Observatoire universitaire de la ville et du développement Durable, université de Lausanne, p. 11-30. Éric Henry, Marie Puybaraud, «Expertises, compétences et gestion de projet en construction durable», dans Véronique Biau, Guy Tapie (dir.), La fabrication de la ville. Métiers et organisation, Marseille, Parenthèses 2009, p. 43-59

2. Jodelle Zetlaoui-Léger (dir.), "L'implication des habitants dans les projets d'éco-quartiers en France évaluation constructive et mise en perspective européenne », LAB'URBA, LET-UMR CNRS LAVUE $n^{\circ} 7218$, CLARA-faculté d'architecture la Cambre-Horta, université libre de Bruxelles et partenaires associés, dans le cadre 
mettre en œuvre de nouveaux dispositifs, a minima informatifs. Un quart des maîtrises d'ouvrage urbaines s'est engagé dans des démarches ambitieuses tendant vers la co-conception, établissant dans ce cas de figure des liens très étroits entre intensité de la participation citoyenne et niveau d'ambition en matière de développement durable.

Or ces évolutions placent les professionnels du cadre bâti, et notamment les architectes, qu'ils exercent en tant qu'assistants à la maîtrise d'ouvrage ou en tant que maîtres d'œuvre, dans des situations d'exercice assez inédites. Elles requestionnent leurs savoirs et leurs savoir-faire dans des systèmes d'acteurs qui se restructurent autour de nouvelles compétences et formes de légitimité. Elles interrogent, plus fondamentalement encore, les pratiques et représentations associées à la démarche de projet, dans des termes qui nous paraissent proches de ceux qu'ont explorés à partir des années 1960 les théoriciens-praticiens anglo-saxons du Design Methods Movement (dorénavant dmm dans le texte). L'objet de cet article est non seulement de donner un aperçu des travaux de ce mouvement assez méconnu en France ${ }^{4}$, mais aussi de montrer en quoi, devant les interrogations que posent actuellement les enjeux du développement durable à l'activité de conception, ils mériteraient d'être (re)découverts.

\section{De nouvelles situations professionnelles pour les architectes}

Alors que la division technique du travail - qui s'est accélérée depuis les années 1970 dans le domaine de l'aménagement et de la construction - a eu tendance à parcelliser et à segmenter les processus de projet, les exigences liées au développement durable incitent à mettre en œuvre des démarches articulant en permanence les différentes dimensions en jeu dans la transformation des espaces urbains. II s'agit de faire interagir sans cesse problématiques sociales, économiques et environnementales comme y invite intrinsèquement ce concept, mais également de mettre en place des dispositifs aussi bien techniques que participatifs permettant d'évaluer les conséquences des solutions envisagées en termes d'appropriation et de gestion ultérieure des espaces aménagés ${ }^{5}$. Le processus de projet ne devrait donc plus être considéré dans une logique strictement séquentielle, de même qu'il ne pourrait plus s'inscrire dans un modèle de relations hiérarchiques entre les acteurs concernés.

Ces deux idées ne sont pas totalement nouvelles : dans le domaine de l'architecture, Michel Conan avait tenté d'introduire à la fin des années 1980 des méthodes dites de "programmation-conception générative » en architecture $^{6}$, mais l'importance prise par les concours sur esquisse dans le cadre de la réforme de l'ingénierie publique avait coupé court aux expérimentations engagées en ce sens.

Dans le domaine des projets urbains complexes, ces préoccupations ont été au cœur du développement d'ingénieries dites « concourantes » depuis le début des années 2000, encadrées par des procédures incitatives apportant une alternative au concours telles que les " marchés de définition simultanés ${ }^{7}$ », et aujourd'hui, le " dialogue compétitif ». Outre les itérations qu'elles sont censées favoriser entre programmation et conception, du Programme "Concertation, décision, environnement » du ministère de l'Écologie, du développement durable et de l'énergie.

3. Yannick Rumpala, Le développement durable ou le gouvernement du changement total, Lormont, Éditions Le Bord de l'eau coll. "Diagnostics », 2010.
4. Peu traduits, à l'exception de certains ouvrages de Christopher Alexander qui est pour autant loin de représenter l'ensemble de ce mouvement, les travaux du DMM restent assez méconnus en France, malgré les tentatives de chercheurs comme Michel Conan ou Robert Prost d'en diffuser certains acquis au début des années 1990.
5. Catherine Charlot-Valdieu, Philippe Outrequin, L'urbanisme durable. Concevoir un écoquartier, Paris, Le Moniteur, 2009.

6. Michel Conan (dir.), Perspectives pour la maîtrise d'ouvrage publique, Plan Construction et Architecture, 1993.

7. J. Zetlaoui-Léger, «Marchés de définition simultanés : un succès à interroger », Urbanisme juillet-août 2002, n³25, p 14-17. 
De la barre à

"l'éco-village ". Quartier des Brichères, Auxerre.

Urbaniste : Serge Renaudie Architecte : Lucien Kroll. (C) Synapse productions
Un des premiers écoquartiers en Europe :

Rieselfeld, Fribourg-en-

Brisgau (Allemagne)

Entre performances

environnementales et

préoccupations d'urbanité.

(C) J. Zetlaoui-Léger
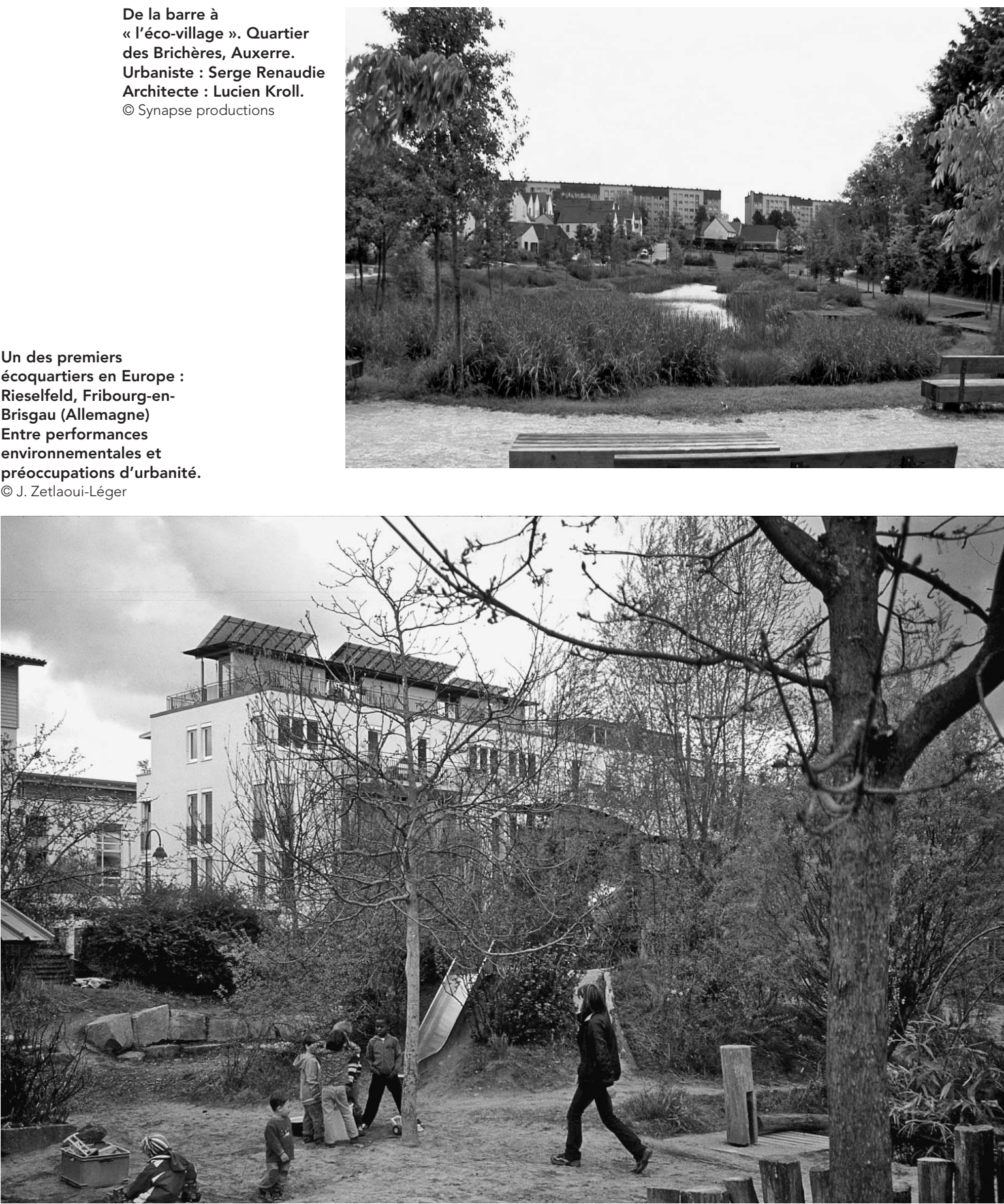
ces démarches visent également à stimuler des nouvelles formes de coopérations entre acteurs. Pour autant, si les consultations réglementaires censées les inciter connaissent un vif succès, les processus, c'est-à-dire la manière dont les éléments de contenu des projets se définissent et les décisions sont prises, peinent à se reconfigurer. Les études et les expertises tendent encore à se juxtaposer, tandis que les habitants prennent rarement le statut de parties prenantes dans les modes de management adoptés ${ }^{8}$.

Les premiers projets d'écoquartiers français témoignent de la prégnance de ces tendances lourdes de I'urbanisme français. Néanmoins, ils révèlent aussi certaines évolutions, surtout quand ils ont été envisagés comme des expérimentations associant par exemple, ambitions fortes en termes de durabilité et de participation citoyenne. Dans ces situations, encore rares mais assez significatives d'évolutions en cours, les habitants se trouvent impliqués dès l'amont et tout au long du processus opérationnel, dans des "démarches intégrées ", c'est-à-dire où les dispositifs participatifs jouent un rôle central dans la fabrication du projet et sont en interaction avec les instances de décision. Souvent très préoccupés par la façon dont se développeront les modes de vie ultérieurs, les habitants mobilisés assurent alors une sorte de "veille programmatique » interrogeant sans cesse et dans une logique itérative les "experts patentés »sur leur approche des problèmes à traiter et sur la portée de leurs solutions. Assistants à maîtres d'ouvrage et concepteurs peuvent se trouver ainsi incités à adopter de nouvelles postures et à faire valoir de nouveaux savoirfaire dans leurs échanges avec la société civile.
Des architectes-urbanistes ou des paysagistes doivent alors faire la preuve de leur capacité à travailler collectivement dans les étapes de programmation-conception, à soumettre différentes intentions d'aménagement et à faire évoluer leur point de vue dans les phases ultérieures. On attend d'eux l'invention de méthodes et de supports de dialogue donnant une véritable clef d'entrée aux habitants pour participer activement à la définition du projet, y compris autour de problématiques parfois très éco-techniques.

Davantage habitués au cours de leur formation initiale, puis dans les consultations auxquelles ils répondent habituellement, à être dans une logique de production de solutions formelles et constructives en défendant solidement un "parti », les architectes ne sont manifestement pas tous très à l'aise dans ces types d'exercice où ils doivent explorer des dimensions programmatiques, et plus précisément d'usages avec les habitants. On peut leur demander de produire des schémas suffisamment ouverts pour être évolutifs, voire plus encore d'aider à la formulation de questions sur leurs propres productions afin de permettre aux habitants d'y apporter des critiques et des contributions ${ }^{9}$. Ils sont ainsi sollicités pour créer les conditions d'un partage collectif de l'acte de conception alors que leur culture professionnelle d'origine les a plutôt incités depuis plusieurs siècles à assumer seuls la « maîtrise de l'œuvre ".

Or ces nouvelles situations d'exercice font assez directement écho aux préoccupations exprimées au cours des années 1960 à 2000, par un ensemble de théoriciens-praticiens anglo-saxons animateurs du Design Methods Movement.
8. Maurice Blanc, "Gouvernance » dans Jean-Marc Stebe, Hervé Marchal (dir.), Traité sur la ville, Paris, PUF, 2009, p. 207-257. Pierre Diméglio, J. Zetlaoui-Léger, « Les rapports ambigus entre politiques et citoyens : le cas du réaménagement du quartier des Halles à Paris », French Politics, Culture \& Society, vol. 25 (2), New-York, 2007, p. 115-140.
9. C'est ce que nous avons par exemple observé pour l'aménagement de l'éco-quartier Danube à Strasbourg ou celui de la Zac Paul Claudel à Amiens. Les travaux de Véronique Biau sur les architectes aujourd'hui engagés sur des projets en auto-promotion, convergent vers ce type d'analyse. Voir « Les architectes de l'habitat participatif, entre militance et compétence », Métropolitiques, 30 janvier 2012. URL : http://www.metropolitiques.eu/ Les-architectes-de-l- habitat.html. 
Les recherches et expérimentations développées pas ce courant très méconnu en France nous paraissent présenter des enseignements tout à fait intéressants sur la façon d'aborder le processus de projet. S'interrogeant sur les fondements de cette activité, elles invitent à considérer celui-ci dans une acception large, en y intégrant des dynamiques évaluatives et participatives, au nom de valeurs et de principes qui sont aujourd'hui ceux du développement durable ${ }^{10}$.

\section{À la recherche du problème}

Le DMM naît au début des années 1960 à la suite d'une série de conférences organisées au Royaume-Uni et aux États-Unis établissant la portée limitée des approches " classiques " du processus de design ${ }^{11}$ face à des projets de plus en plus complexes à réaliser. Critiquant le caractère trop intuitif et informel des démarches traditionnelles, la préoccupation des précurseurs du DMM est de refonder le processus de projet à partir d'une réflexion sur la programmation comme système de gestion de données permettant de mieux préparer le travail de mise en forme. Ils se fondent pour cela sur des premières expériences programmatiques en architecture ainsi que sur des méthodes et outils issus de recherches menées par des agences gouvernementales dans le domaine militaire durant la Seconde Guerre mondiale et dans celui de la conquête spatiale depuis les années $1950^{12}$.

La programmation est perçue comme une action devant exprimer les tenants et aboutissants d'un projet dans son ensemble. Elle ne consiste pas seulement à rassembler des informations sur des "besoins", des activités ou des contraintes constructives avant de " composer", comme c'était le cas avec le modèle cartésien " analyse/synthèse ", mais elle suppose une organisation et une hiérarchisation des données pour guider des décisions, en définissant et en articulant «fins et moyens ». On retrouve dans cette approche les principes des démarches d'action rationnelle utilisées dans le domaine du management des organisations, science qui se développe alors aux États-Unis comme en France et qui conduit l'un de ses spécialistes, Herbert Simon, à s'intéresser à la conception architecturale dans des termes qui auront une influence importante sur les protagonistes du DMM.

À l'occasion de ses travaux sur les mécanismes de la décision dans les administrations, Simon est amené à considérer l'être humain comme un système de traitement d'informations. Ne parvenant pas à expliquer les prises de décision par une approche déterministe, ni par le modèle économique de la « rationalité objective et parfaite ", il invente les concepts de " rationalité limitée " et de "satisfecit » (solution à peu près satisfaisante) qu'il intègre dans une théorie générale sur la résolution de problèmes que l'exemple du processus de conception spatiale l'aide à élaborer. Au même titre que d'autres domaines comme la médecine ou la gestion des organisations dans lesquels une grande quantité d'informations doit être traitée par des systèmes de symboles ou de représentations, Simon considère le processus de design comme une "science de l'artificiel " consistant à transformer une situation existante non désirée, en une situation aux propriétés souhaitées par la fabrication "d'artefacts ». Il présente
10. Guillaume Faburel, Camille Roché, Les valeurs et principes de l'aménagement durable. Analyse et perspectives par et pour les écoquartiers en France, Aménités, MEEDDTL, AD4, 2012.

11. Pour les théoriciens-praticiens du DMM, ce terme renvoie à la fois à la formulation d'un dessein général et à un travail de formalisation.
12. Mickey A. Palmer, The Architect's Guide to Facility Programming, American Institute of Architects, 1981 
cette entreprise comme une démarche au cours de laquelle seront tenues pour bonnes ou satisfaisantes des solutions qui ne seront pas forcément optimales ${ }^{13}$.

À l'instar d'Herbert Simon, les théoriciens-praticiens du DMM vont utiliser cette métaphore du « problème » pour évoquer la nature du travail qu'ont à mener les architectes. Tout en critiquant pour certains d'entre eux l'approche relativement "mécanique " proposée par Simon dans sa vision du processus de conception architecturale ${ }^{14}$, ils développent au cours des quatre décennies suivantes différentes réflexions sur la notion de "problème » ainsi que sur le positionnement et les objectifs de la démarche programmatique dans le processus de design. Leurs travaux, très influencés par la philosophie pragmatique anglo-saxonne, aboutissent à la définition de méthodes et d'outils spécifiques pour des échelles d'intervention allant du bâtiment au quartier.

Dans un ouvrage faisant toujours référence, Nigel Cross $^{15}$ a rassemblé en 1984 un ensemble de textes fondateurs et de témoignages mettant en exergue deux générations de travaux sur les méthodologies du design. La première qui se structure entre 1962 et 1967 tente de systématiser le processus de conception à partir des apports des méthodes et des techniques issues de recherches sur le management et les systèmes de prise de décision. Ces réflexions se caractérisent par la recherche d'un traitement rigoureux et logique des données à partir d'analyses systémiques empruntant aux théories mathématiques et aux sciences de l'information et de la communication.

ChristopherAlexander, mathématicien etarchitecte qui débuta ses travaux sur la programmation au Royaume-Uni avant de partir à la fin des années 1950 aux États-Unis pour enseigner à Berkeley fut l'un des précurseurs de ce type d'approche. Dans ses recherches au début des années 1960, il tente de mettre au point une démarche de type algorithmique empruntant sa logique et son formalisme à la théorie des graphes et au langage informatique, sans renoncer à l'approche traditionnelle analyse/synthèse qu'il combine avec une démarche en termes de définition / résolution des problèmes ${ }^{16}$. II propose d'aborder l'aménagement d'une ville comme la réalisation d'un bâtiment par un travail de décomposition du problème en une succession d'énoncés élémentaires qui représentent les exigences spécifiques du contexte par rapport au projet. Ces composants élémentaires se trouvent mutuellement disjoints ou regroupés dans un même sous-ensemble en fonction de leur proximité ou de leur indépendance respective, selon un modèle hiérarchique en arborescence qui exprime les différentes propriétés que devra avoir la forme (phase d'analyse). Alexander invite ensuite à trouver des réponses partielles formelles pour chacun de ces énoncés, puis sous-systèmes d'énoncés, la résultante de ce travail (synthèse) devant permettre in fine, de construire la solution architecturale globale recherchée.

La ville $n^{\prime}$ est pas un arbre ${ }^{17}$, selon Alexander. Les critiques qui lui ont été adressées et selon lesquelles, d'une part, la solution à un niveau donné est susceptible de remettre en question celles adoptées à un niveau inférieur, et d'autre part, l'objet final en architecture ne peut être la traduction d'une logique fonctionnelle hiérarchique. Abandonnant son projet scientiste qu'il considèrera lui-même comme déshumanisant, il
13. Cette réflexion est développée dans un ouvrage paru en 1969 et traduit par Jean-Louis Lemoigne en première édition en France en 1974, Les sciences de l'artificiel, Gallimard coll. «Folio essais », 2004.

14. Pour Donald A. Schön, auteur d'un travail critique de l'approche d'Herbert Simon, la conception architecturale ne peut être considérée comme une activité d'application mécanique d'une procédure. Pour lui, les concepteurs sont amenés à rencontrer des situations inattendues dans la construction de leur problématique qui les conduisent, dans une posture réflexive et critique, à réorienter leur action en cours de procès de design. Donald. A. Schön, The Reflective Pratictionner, Londres, Temple Smith, 1983.

15. Nigel Cross, Developments in Design Methodology, Chichester, John Wiley and Sons, 1984.
16. Christopher Alexander, De la synthèse à la forme, essai, Paris, Dunod, 1971, trad. fr. de Notes on the Synthesis of Form, Harvard University Press, 1964.

17. Ch. Alexander, "A City is not a Tree", Design, $n^{\circ} 206$, février 1966, p. 46-55. Dans cet article, Alexander explique le succès du fonctionnalisme et ses dangers. 
privilégiera alors des méthodes de programmationconception participatives à partir d'un outil de dialogue et d'invention, le pattern language (le langage des modèles) permettant d'associer des problèmes-clés d'aménagement de l'espace à des formes d'organisation spatiales ${ }^{18}$. Or Christopher Alexander reste le principal représentant du DMM connu en France grâce à la traduction de deux de ses principaux ouvrages, De la synthèse à la forme et Une expérience d'urbanisme démocratique.

Moins célèbre qu'Alexander, William Peña est pourtant considéré dans les pays anglo-saxons comme un théoricien majeur des processus de Design. II écrit un premier texte sur ce sujet dès 195919, sur la base d'expériences qu'il a conduites à partir de la fin des années 1940 au sein de son bureau d'études au Texas. Si l'approche de Peña est analytique et séquentielle comme celle d'Alexander dans ses premiers travaux, elle est moins abstraite et davantage inscrite dans une logique compréhensive de recherche des enjeux et des conditions de réalisation des intentions d'un projet. Souhaitant manifestement se distinguer de ce dernier, Peña affirme d'ailleurs que "la programmation est un processus heuristique et non un algorithme ${ }^{20}$ ». La notion de problème qu'il adopte ne revêt pas non plus la même signification que celle adoptée par Alexander : elle ne désigne pas la question initiale donnée à l'architecte. Peña estime qu'au départ, les limites du problème ne sont pas bien connues et sont à déterminer dans le cadre du processus de programmation. La démarche ne garantirait toutefois pas forcément la définition complète du problème, mais permettrait de réduire le nombre d'incertitudes. Pour définir le problème, il propose cinq étapes de programmation :

« 1 : Établir les buts : que veut réaliser le client et pourquoi?

2 : Collecter les faits : de quoi s'agit-il ?

3 : Découvrir les concepts : comment le client veut-il réaliser ces buts?

4 : Besoins : combien d'argent, d'espace et quelle qualité ?

5 : Définir le problème : quelles sont les conditions significatives et les directions générales que le bâtiment doit prendre ?21"

La notion de besoin intervient chez Peña avec un sens différent de celui qui lui est donné dans les approches fonctionnalistes traditionnelles. Les besoins n'existent pas à priori, et ne sont pas définis de façon universelle pour un type d'espace à réaliser; ils sont le résultat d'un processus de construction où ils doivent être considérés comme des moyens au service d'objectifs particuliers. Peña mentionne par ailleurs que l'ordre des tâches qu'il énonce n'est pas forcément celui qui sera scrupuleusement suivi dans la pratique. Néanmoins, il affirme que la cinquième étape ne peut être réalisée qu'à l'issue des quatre premières, c'est-à-dire après avoir rassemblé toute l'information nécessaire à définir le problème. La formulation la plus complète possible du problème marque la fin du travail de programmation et annonce sa résolution formelle; si la programmation et la conception sont envisagées comme faisant partie d'une démarche générale de design, en revanche ces deux activités sont nettement dissociées et présentées comme étant de nature très différente.
18. Le Pattern Language est pour Alexander " un système fini de règles qu'une personne peut utiliser pour générer une variété infinie de bâtiments différents ". D'après The Timeless Way of Building, New York, Oxford University Press, 1979, p. 191.
19. William Peña, Caudill William, «Architectural Analysis. Prelude to Good

Design », Architectural Record, vol. $125 n^{\circ} 5$, mai 1959, p. 178-182.

20. W. Peña, Steven A. Parshall, Problem Seeking. An Architectural Programming Primer, CBI Publishing Barton [1969], rééd. 1987, p. 9
21. Ibid. p. 8 
Tout en proposant des méthodes spécifiques, Alexander et Peña séparent donc nettement programmation et conception dans leurs premiers travaux. Ils n'hésitent pas aussi à croire - comme une sorte de postulat - à la possibilité de définir et de résoudre un problème de design dans toutes ses dimensions.

\section{L'influence des Environmental Studies}

À partir de la fin des années 1960, la pertinence de ces démarches systématiques et séquentielles est contestée. Sont remises en question l'ambition de parvenir à une appréhension exhaustive des problèmes de design ${ }^{22}$ tout comme le caractère "objectif " et inductif du processus de design. À l'occasion d'une conférence organisée en 1968 au Massachussets Institute of Technology, mit, apparaît ainsi un nouveau courant au sein du DMM, établissant un lien étroit avec un champ de recherches émergent, celui des Environmental Studies. Ce dernier se construit au cours de cette période à l'initiative d'un ensemble de théoriciens, proches de la deuxième école de Chicago et issus de différentes disciplines des sciences humaines et sociales ${ }^{23}$. Ils partagent un même objet de réflexion : les relations entre I'homme et son milieu de vie. Au-delà des spécificités de leurs interprétations, ils proposent d'aborder la notion d'environnement en y intégrant le naturel et le bâti, les phénomènes sociaux et psychologiques, dans une perspective globale et écologique s'intéressant aux impacts, aux interactions et aux interdépendances entre ces dimensions. Alors qu'ils essaient eux-mêmes de tisser des liens avec le champ de l'aménagement ${ }^{24}$, leurs travaux rejoignent les préoccupations des tenants d'une approche " humaniste " des problèmes de conception au sein du DMM, qui mettent en doute la capacité de la pensée positiviste et des évolutions technologiques à produire un monde meilleur. Très soucieuse de prendre en considération les relations de I'homme à son environnement au cours du processus de conception puis une fois le bâtiment livré, cette seconde génération de théoriciens-praticiens du DMM va engager tout un ensemble de recherches et d'expérimentations sur l'évaluation et sur la place des habitants, utilisateurs usagers, dans la production du cadre bâti.

John Zeisel, un sociologue de l'université d'Harvard est l'un des initiateurs de ce second courant. Travaillant sur des institutions qui hébergent des patients atteints de pathologies mentales et sur des établissements scolaires, il est particulièrement préoccupé par les relations entre comportements et qualité du cadre de vie. S'il admet que des idées préconçues, subjectives s'exprimant par un premier parti pris formel, engagent le processus de design ${ }^{25}$, il attache une grande importance à la prise en compte de dimensions psychosociales dans le processus de conception. II s'intéresse alors à l'évaluation comme un moyen pour l'architecte d'être conscient des conséquences des idées qu'il avance sur le rapport homme-environnement et comme un support au dialogue avec son client et les futurs usagers ${ }^{26}$.

En rupture avec l'ambition qu'avait eue un certain nombre de chercheurs du DMM dans les années 1960 d'aborder de façon totalement « objective » et exhaustive les problèmes de conception, les travaux de Zeisel se distinguent également des approches systématiques de la première génération du DMM en supposant
22. En 1973, Horst W. J. Rittel et Melvin M. Webber dans un article qui fera référence, démontrent que les problèmes d'aménagement et de constructions sont " pernicieux » (wicked-problem) ou « mal structurés " (ill-structured) car relevant de contextes sociétaux et sociaux impossibles à appréhender dans leur totalité "Dilemnas in a General Theory of Planning », Policy Sciences vol. 4 n² 2, 1973, p.155-169.
23. Erwin Goffman, Edward T. Hall ou encore Amos Rapoport seront des références majeures pour le second courant du DMM.

24. Voir à ce sujet le rapport d'étude de Pierre Teasdale, Évaluation et programmation en aménagement, Faculté de l'aménagement de l'université de Montréal, 1979.
25. II s'appuie en cela sur les travaux de Jane Darke, "The Primary Generator and the Design Process », Design Studies vol. 1 $n^{\circ} 1,1979$, p. 36-44.

26. John Zeisel, Inquiry by Design: Tools for Environment-Behavior Research, Cambridge University Press, 1984. 
que les problèmes se découvrent progressivement par un processus itératif de mise à l'épreuve des solutions proposées. Pour Zeisel, lorsque le designer appréhende un problème, il crée une image mentale qui va permettre d'engager le processus de design en étant porteur d'un principe général de solution spatiale. Il doit être en mesure de l'exprimer de telle façon que celle-ci puisse être soumise à vérification et éventuellement modifiée. Le travail d'explicitation est ici fondamental souligne-t-il, designers present not images themselves but the implications of images ${ }^{27}$. La représentation de la solution doit être suffisamment clarifiée par l'énoncé des intentions programmatiques - envisagées comme hypothèses faites sur des modalités d'usage de l'espace par exemple - pour pouvoir être confrontée à un ensemble d'objectifs et de critères à satisfaire. Le concepteur doit donc être en mesure de clarifier les enjeux d'usages sous-jacents aux solutions formelles qu'il propose parfois sur des bases intuitives, pour les soumettre à analyse critique. L'activité d'évaluation a en fait un double rôle, d'appréciation de la qualité des solutions envisagées et de génération de propositions alternatives de nouvelles "images " : Testing is a feed-back and a feed-foward process ${ }^{28}$. Le développement d'une solution prend fin lorsque l'organisation cliente estime que celle-ci est « satisfaisante " (voir Simon) et non pas lorsqu'il y a une adaptation " idéale » de la forme au problème à traiter comme le suggéraient les théoriciens de la première génération.

La situation qu'évoque Zeisel dans son ouvrage Inquiry by Design interpelle celles que rencontrent des concepteurs aujourd'hui impliqués dans des dispositifs participatifs. Dans ces cas de figure, on constate en effet que si les habitants se trouvent seulement confrontés à des images ne renvoyant qu'à des solutions spatiales, les débats peinent à s'engager ou deviennent rapidement houleux, surtout si le projet est présenté pour une première fois publiquement à un stade assez avancé de formalisation. Il s'avère en effet fondamental pour que le dialogue parvienne à s'instaurer de façon apaisée et constructive, de permettre aux habitants d'appréhender voire d'évaluer les propositions d'aménagement à travers des questionnements d'ordre notamment programmatiques. Le concepteur ne peut rester dans I'implicite : il lui faut objectiver les intentions d'usages du projet qu'il soumet, ou aider les habitants, par la production de supports adaptés, à exprimer leurs attentes sur les usages des lieux à aménager. Cette condition apparaît d'autant plus nécessaire lorsque l'opération est soumise à de fortes ambitions environnementales susceptibles de modifier structurellement le cadre de vie futur. Collectifs d'habitants ou associations mobilisés se montrent alors particulièrement soucieux de comprendre voire de contribuer à la définition des nouveaux modes d'habiter induits par les espaces préfigurés.

\section{De «l'évaluation performantielle» à l'Universal Design}

Les précurseurs du DMM avaient très rapidement intégré la pratique de l'évaluation dans leurs réflexions sur la nature du processus de design. Au départ, celle-ci intervenait néanmoins surtout à la fin de la phase de conception. Au cours des années 1970, émerge à l'initia-

27. Ibid. p. 8.

28. Id. p. 9. 
tive des théoriciens de la seconde génération du DMM, toute une série de nouveaux travaux basés sur des recherches-actions s'intéressant aux méthodes et aux outils d'évaluation dans le domaine de la production des édifices qui insistent sur la nécessaire systématisation de cette pratique. Celle-ci apparaît comme un processus restant actif durant toute l'élaboration du projet et après la livraison du bâtiment. Quatre grands objectifs sont associés à l'évaluation. Ils correspondent à différentes phases inscrites dans ce qui est désigné par une analogie avec le monde industriel, le « cycle de vie » d'un bâtiment $^{29}$ :

- au cours du processus d'élaboration du projet, pour tester la façon dont celui-ci évolue en répondant bien aux objectifs qui lui sont conférés ;
- une fois le projet construit, au bout de deux années de fonctionnement de préférence, pour vérifier si celui-ci répond bien à ses objectifs ;

- après quelques années d'utilisation, pour recueillir des informations qui pourront servir à la réalisation d'un nouveau bâtiment :

- dans le cas où l'équipement pose de grands problèmes, pour remettre en question des processus de programmation-conception ou du type même de politique ou de solution constructive.

Les trois derniers stades donnent lieu à des PostConstruction Evaluations (PCE) ou à des Post-Occupancy Evaluations (POE). Les PCE supposent de réaliser des mesures qui permettent d'apprécier les niveaux de performances techniques et économiques d'un bâtiment.
L'évaluation des performances des bâtiments : une démarche intégrée, d'après Wolfgang F. E. Preiser. " Toward Universal Design Evaluation ", Universal Design Handbook, New York, Mc-Graw-Hill, 2001, p. 9-13.

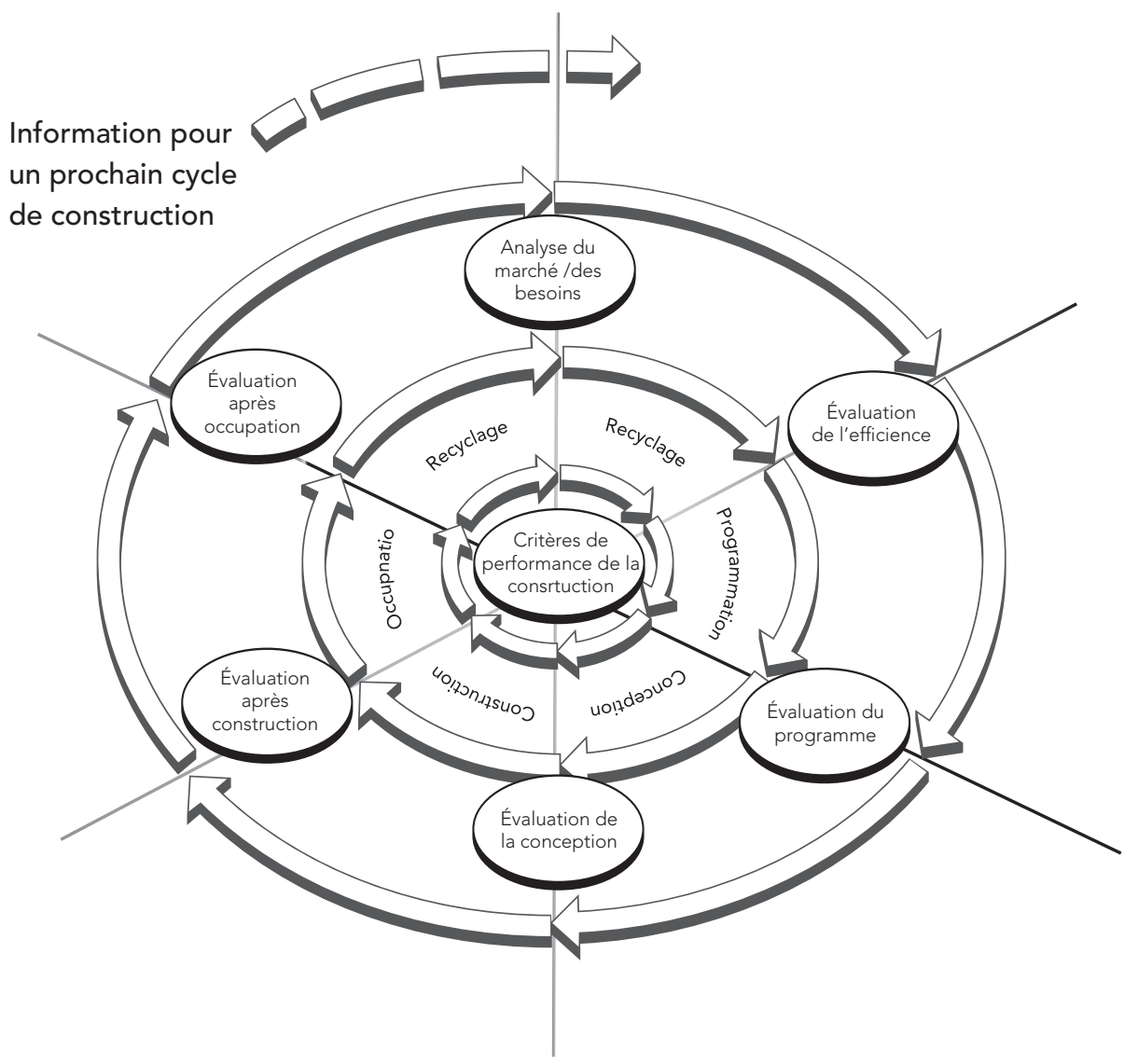


Ces démarches reposent sur une représentation de la programmation architecturale exprimée selon une logique dite " performantielle ${ }^{30}$ ». La programmation y est présentée comme une activité qui permet à une « organisation cliente » de déterminer des attentes vis-à-vis d'un bâtiment afin d'en améliorer la qualité et l'efficience. La rédaction d'un programme dans des termes « performantiels » permet à certains de considérer ce document à la fois comme un outil permettant de vérifier l'adéquation des solutions constructives aux intentions de la maîtrise d'ouvrage, comme un outil d'évaluation périodique " post-occupation » et comme un manuel d'utilisation du bâtiment pour ses occupants. C'est l'expérience que mène l'architecte Jay Farbstein dans le cadre de la réalisation d'un centre d'éducation fermé pour adolescents : " Habituellement, le programme ne trouve pas d'autre application une fois le projet achevé. Dans ce cas cependant, le programme a été à l'origine d'un processus où ses dimensions socio-spatiales ont pu être enrichies pendant le projet et testées après occupation pendant une période continue. Il a permis de constituer un manuel d'utilisation ${ }^{31}$. [...] Lors de la mise en service du bâtiment, l'équipe de direction et les personnels du centre ont reçu (ce) manuel pour les aider à comprendre et à faire fonctionner leur bâtiment. Ce document contenait une partie concernant les hypothèses qu'avaient faites les programmateurs et les concepteurs sur la façon dont le bâtiment pourrait fonctionner et évoluer ${ }^{32}$ ».

Le manuel a ensuite été utilisé pour définir une méthode générale d'évaluation et définir en particulier une critériologie. Jay Farbstein considère qu'il est de la responsabilité de l'équipe de programmation- conception d'accompagner les utilisateurs et les usagers dans l'occupation d'un nouvel équipement, l'évaluation faisant selon eux partie de l'activité de programmation.

Ces expériences qui suggèrent d'intégrer dans la réflexion programmatique et à travers les outils qu'elle produit, les modalités d'évaluation et d'appropriation future d'un bâtiment, mériteraient d'être portées à la connaissance des professionnels de l'aménagement et de la construction en France. Les "livrets verts" des " éco-gestes " visant à faciliter l'installation des résidents dans des espaces à hautes performances environnementales se multiplient en effet. Ces derniers sont élaborés à posteriori, principalement dans une logique "d'adaptation » des occupants à de nouveaux modes d'habiter organisés autour d'éco-techniques encore rarement mises au point avec le concours de leurs usagers. Par ailleurs, l'évaluation d'usages ex-post des réalisations est encore loin d'être systématiquement prévue par les maîtres d'ouvrage dans les projets d'aménagement actuels.

Les démarches d'évaluation engagées par des organisations publiques et privées dans les pays anglo-saxons à partir des années 1970 traduisaient des soucis d'efficacité économique et financière dans une approche qu'on pourrait qualifier de « coût global » du projet. Mais elles se sont mises à intégrer peu à peu aussi des dimensions sociales et psychologiques dans le cadre d'approches environnementales diffusées par des théoricienspraticiens de la seconde génération tels que Wolfgang Preiser. Ces derniers vont chercher à promouvoir une pratique de l'évaluation prenant en considération ces dimensions à travers une réflexion programmatique,
29. Donna P. Duerk, Architectural

Programming. Information Management for

Design, John Wiley and Sons, 1993.
30. À noter que cette expression avait été déjà utilisée par Peña à la fin des années 1960.

31. Traduit de Jay D. Farbstein, « Using the Program. Applications for Design, Occupancy, and Evaluation », dans Wolfgang F. E. Preiser, Programming Built Environment, New York, Van Nostrand Reinhold Compagny, 1992, p. 13.

32. Ibid. p. 13-29. 
porteuse de problématiques d'usages et de perception sensorielle, qui reste active tout au long du processus de projet. Tandis que nombre des travaux menés sur I'évaluation des bâtiments dans les années 1980 et 1990 dans les pays anglo-saxons poursuivent l'ambition de mesurer et de quantifier tous les types de rapports que les individus peuvent entretenir avec leur environnement afin de constituer des bases de références, voire, plus largement, des banques de données à l'échelle internationale, Wolfgang Preiser insiste sur la nécessité de prendre en considération des critères autres que techniques ${ }^{33}$ pour apprécier la façon dont est perçu un environnement bâti ainsi que ses effets sur les usagers. II met également en garde contre la transposition mécanique de normes pour définir les performances recherchées et préconise que ces dernières soient établies à partir des « standards recensés " pour des bâtiments de même type, ainsi que par rapport aux objectifs de l'organisation cliente et aux attentes des usagers.

Attaché à la prise en compte de la spécificité de chaque citoyen dans sa relation à l'espace et aux organisations sociales, Preiser donne à partir de la fin des années 1990 un nouveau cadre conceptuel à son approche de l'évaluation, I'Universal Design ${ }^{34}$. II défend avec d'autres chercheurs-praticiens au sein du Center for Universal Design qu'il a fondé en 1997, une approche du processus de design favorisant l'égalité et la justice sociale dans l'accès des personnes, quelle que soit leur condition, aux espaces et aux services publics.

Dépassant la préoccupation de produire de nouvelles normes à laquelle se trouve souvent limitée par exemple, la question de l'accessibilité en architecture et en aménagement, Preiser et ses collègues font la promotion de démarches d'évaluations participatives, intégrant des dimensions sociales et sociétales dans l'appréciation de la qualité environnementale, selon des modalités proches de celles qui structurent aujourd'hui les enjeux du développement durable.

\section{Portée heuristique et démocratique des démarches participatives}

Pour tout un courant de chercheurs-praticiens du DMM qui articulent étroitement leurs travaux aux Environment Studies, la compréhension des valeurs sociales et des activités humaines prend au cours des années 1970 une dimension centrale dans leur approche du processus de design. Ils estiment que les rapides évolutions de la technologie, les transformations des processus de travail et les attentes croissantes des consommateurs vis-à-vis de leur cadre de vie ont aggravé les problèmes créés par une société déjà fragmentée ${ }^{35}$. Ils investissent de ce fait la programmation d'une mission sociale tout en reconnaissant aux usagers-citoyens une expertise complémentaire à celles des planificateurs et des architectes.

Alors que la question de la résolution des problèmes de design avait tendance à être abordée essentiellement sur le mode de la réflexion créative, Henry Sanoff, architecte, professeur à la North Carolina State University, estime qu'elle est aussi fondée sur la résolution de conflits d'intérêts. Par ailleurs, tandis que chez les premiers théoriciens du DMM, le processus de design était centré sur la relation client-designer, il introduit la notion de groupes d'usagers. Ces groupes ne sont pas
33. Comme la santé, la sûreté et la sécurité / la fonctionnalité, l'efficience et l'impact sur la productivité au travail / les effets psychologiques, sociaux, culturels et esthétiques induits.
34. Le Center for Universal Design est un organisme universitaire d'informations, de recherches, d'expérimentations faisant la promotion de cette approche du design orientée vers la lutte contre toutes les formes de discriminations. http://www.ncsu. edu/project/design-projects/udi/.
35. Wolfgang F. E. Preiser, « Responding to the changing context of environment design ", Facility Programming. Methods and Applications, Stroudsburg, Dowden, Hutchinson and Ross., 1978. 
seulement considérés comme des ressources d'informations mais comme des instances exprimant des valeurs et contribuant à la décision. Différents rôles leur sont attribués et plusieurs techniques de conduite leur sont appliquées selon le moment où ils interviennent.

Pour Sanoff, la participation produit « une source de témoignages et d'informations au sujet du contexte local, des besoins et des attitudes, permettant ainsi d'améliorer l'efficacité du processus de décision et d'utiliser au mieux les ressources dont dispose une collectivité ${ }^{36}$ ". Elle enrichit aussi les connaissances du designer par des formes d'expertises pratiques. Pour Sanoff, ce dernier doit, abandonner sa posture traditionnelle qui le conduit à affirmer voire à imposer ses propres intentions, pour aider les gens à déterminer ensemble ce qu'ils souhaitent et ce qui pourra être bénéfique pour les uns et les autres. Le professionnel ne renonce pas à son statut de spécialiste des questions de design dans les recommandations qu'il peut prodiguer et dans sa capacité à montrer les conséquences des choix envisagés, mais joue aussi un rôle de facilitateur d'expressions. II doit dès lors accepter la dimension collective de l'œuvre produite.

Un autre enjeu de la participation est pour Sanoff d'ordre social et politique. II s'exprimerait simultanément à l'échelle de la société, des groupes et des individus. La participation ferait progresser la démocratie en élevant le niveau de conscience sociale de la « communauté de vie ». Elle réduirait un sentiment d'anonymat souvent éprouvé par les plus démunis vis-à-vis des autorités administratives, et conduirait à se sentir davantage concerné par la gestion de la " communauté " de vie et par l'avenir de celle-ci. Elle contribuerait à faire en sorte qu'une diversité

36. Henry Sanoff, Integrating Programming,

Evaluation and Participation in Design: A

Theory Z Approach, Aldershot, Avebury,

1992, p. 79 de valeurs sociales soit prise en considération lors de la définition d'une action. Elle serait «le seul moyen de tenir compte de la pluralité des besoins en jeu dans un projet dont certains sont souvent ignorés par les grandes organisations et leur système bureaucratique ${ }^{37}$ ". La mise en œuvre de dispositifs participatifs suppose selon Sanoff une objectivation des principes de la démarche et la mise en place de systèmes de communication permettant aux différents acteurs concernés de s'y impliquer. La programmation est définie comme une démarche de " management par objectifs».

Dès le début, ces derniers doivent être explicités, discutés et partagés même s'ils peuvent évoluer par la suite. L'une des clefs de la réussite de ce type d'entreprise est, pour Sanoff, que les participants puissent apprécier le chemin accompli et le rôle qu'ils ont joué dans l'élaboration du projet. Une autre est la transparence du processus de décision. On trouvera ainsi dans les idées développées par Henri Sanoff, mais aussi par John Zeisel ou par Wolfgang Preiser, une proximité évidente avec celles défendues à partir des années 1980 par les militants des démarches " délibératives » dans la planification urbaine aux États-Unis. Ces principes ont commencé à être introduits en France dans les domaines de l'aménagement territorial et de l'environnement depuis le début des années 2000, mais peinent encore à l'être en urbanisme et en architecture ${ }^{38}$.

Au-delà de leurs différences d'approches, les protagonistes du Design Methods Movement invitent à considérer le processus de projet comme une démarche de définition et de résolution de problèmes. Ils appréhendent de ce fait l'activité de programmation comme une partie
37. Ibid. p. 55

38. Marie-Hélène Bacqué, Mario Gauthier, "Participation, urbanisme et études urbaines ", Participations $n^{\circ} 1,2011$, p. $36-66$. 
intégrante de celui-ci et comme un moyen de le mener à bien à partir de méthodes réflexives et négociées. Loin de s'apparenter à une fonction technocratique et normative déconnectée de la démarche de conception à laquelle elle est souvent assimilée en France ${ }^{39}$, on remarquera que la programmation est envisagée par les théoriciens du DMM liés aux Environmental Studies comme une méthode de questionnement, un support à l'invention basé sur des principes d'évaluation, de délibération collective et de participation citoyenne, aujourd'hui reconnus comme fondamentaux pour favoriser la dimension « durable » des projets.

En France, la question du développement durable a d'abord été abordée dans le domaine de la construction sous un angle économique et technique. L'attention s'est focalisée sur des objectifs à atteindre exprimés à l'aide de cibles dites de " haute qualité environnementale » visant à limiter, surtout par le choix de matériaux et de systèmes constructifs adaptés, toutes sortes d'effets indésirables pour l'homme et son milieu naturel. Ces exigences ont eu tendance à être formulées de façon normative et à partir des phases de maîtrise d'œuvre. S'est ainsi opérée une sorte de dissociation entre programme "fonctionnel » et programme "écotechnique " accentuée conjointement par l'intervention successive de différents experts et par les modes de dévolution des marchés de maîtrise d'œuvre. Dans des pays comme la Hollande d'une part, pionnière dans le domaine des constructions durables en Europe et au Royaume-Uni d'autre part, les connaissances relatives aux dimensions techniques, économiques et d'usages ont plutôt été mobilisées dans une dynamique "d'hybridation » à travers une articulation forte entre programmation et conception ${ }^{40}$.

Les décalages aujourd'hui observés en France entre usages attendus et usages effectifs des dispositifs éco-techniques mis en place ${ }^{41}$, tout comme les enjeux associés à la démocratisation de l'action publique territorialisée, suscitent des remises en question dans la définition des ingénieries de projet. Ils appellent à des démarches plus itératives et participatives, visant à intégrer les expertises y compris habitantes plutôt qu'à les juxtaposer ${ }^{42}$.

II s'agit d'appréhender un projet comme une démarche toujours active, dont la portée et les modalités d'appropriation sont cycliquement à réinterroger par la programmation et l'évaluation. Les travaux du DMM peuvent constituer des sources d'inspirations fructueuses pour les architectes dans cette perspective. Ils suggèrent d'adopter de nouvelles postures vis-à-vis de ces activités jusqu'à présent surtout perçues comme des dispositifs réglementaires et des contraintes à la création. Ils apportent aussi des éclairages stimulants sur la façon d'appréhender le processus de projet comme un objet d'investigation en soi ou comme un terrain d'expérimentation s'inscrivant dans le cadre d'une démarche scientifique.
39. J. Zetlaoui-Léger, « La programmation architecturale et urbaine : émergence et évolutions d'une fonction ", Les Cahiers de la recherche architecturale et urbaine, Paris, Éditions du Patrimoine $n^{\circ} 24 / 25$, décembre 2009 , p. $143-158$
40. Éric Henry, Marie Puybaraud, op. cit. note 1.

41. Vincent Renauld, « Les conceptions techniques innovantes face aux règles d'usage des habitants : enquête sur un bâtiment écologique emblématique de l'écoquartier $\mathrm{De}$ Bonne à Grenoble », Contribution scientifique et technique sur la notion d'appropriation dans les opérations d'aménagements urbains durables, Union sociale de l'habitat -MEDDTL/ AD4, 2012, p. 34-38.
42. Comme le montrent les réflexions et les dispositifs de soutien aux collectivités territoriales qui accompagnent depuis 2009 la procédure de labellisation des «ÉcoQuartiers » mise en place par le ministère de l'Écologie, du développement durable et de l'énergie. 